\title{
Conditioned blocking and schizophrenia: a replication and study of the role of symptoms, age, onset-age of psychosis and illness-duration.
}

\author{
Stefan Bender ${ }^{1}$, Bernhard Müller $^{2}$, Robert D. Oades ${ }^{2 * *}$, Gudrun Sartory $^{3}$, \\ 2001 Schizophrenia Research, 49, 157-170
}

1. University Clinic for Psychiatry and Psychotherapy, Virchowstr. 174, 45147 Essen, Germany.

2. University Clinic for Child and Adolescent Psychiatry and Psychotherapy, Virchowstr. 174, 45147 Essen, Germany *.

3. Department for Clinical Psychology, University of Wuppertal, Max-Horkheimer-Str. 20, 42097 Wuppertal, Germany.

** Address for correspondence:

\begin{abstract}
:
Measures of selective attention processing like latent inhibition (LI) and conditioned blocking (CB) are disturbed in some patients with schizophrenia. (LI is the delay in learning about the associations of a stimulus that has been associated with no event [vs. de novo learning]; $C B$ is the delay in learning the associations of a stimulus-component when the other component has already started to acquire these associations.) We proposed, 1) to replicate the reported decreases of $C B$ in patients without paranoid-hallucinatory symptoms, 2) to see if CB depends on the age of illness-onset and its duration, as reported for LI. We studied 101 young and old, acute and chronically ill patients with schizophrenia, of whom 62 learned a modified 'mouse-in-house' CB task, and compared them with 62 healthy controls matched for age, education and socio-economic background. CB was more evident in patients with a diagnosis of paranoid schizophrenia than other subtypes. An unusual persistence of high $\mathrm{CB}$ scores through testing was associated with productive symptoms (including positive thought disorder). Reduced $\mathrm{CB}$ related to the expression of a) Schneider's first rank symptoms of ideas-of-reference and $b$ ) to negative symptoms like poor rapport and poor attention. $\mathrm{CB}$ was less evident in the older patients and those with an earlier illness-onset. In contrast to the similar $\mathrm{LI}$ test of selective attention $\mathrm{CB}$ is found in patients with paranoid schizophrenia and its expression is not related closely to illness-duration. This implies that the two tests reflect the activity of different underlying processes. We suggest reduced $C B$ on initial test-trials in nonparanoid schizophrenia reflects the unusual persistence of controlled information processing strategies that would normally become automatic during conditioning. In contrast continued CB during testing reflects an unusual persistence of automatic processing strategies.
\end{abstract}

\section{Key Words:}

Attention; Blocking; Illness-duration; Onset; Nonparanoid schizophrenia; Paranoid; $\quad$ Thought disorder

\section{Acknowledgements:}

The authors are grateful to Andrea Czwenk, Angela Dollberg, Isabelle Eisenbeis, Luigi Fierro, Claudia Heeper, Arndt Hesse, Renate Thienel and Dr. J. Wolstein for their friendly and helpful assistance in assembling the clinical data and to the clinic directors Professor M. Gastpar and Professor C. Eggers for their interest and support throughout. We also thank the Deutsche Forschungsgemeinschaft for financial support (DFG OA/4-1 \& 2). 


\section{Introduction}

"Studies comparing a schizophrenia group with a comparison group often show differences without clarifying whether the difference relates only to a subgroup of the subjects". This critical comment from Carpenter and colleagues (1999) can hardly be more poignantly and appropriately applied than to a consideration of how a patient with schizophrenia selects information from what $s /$ he perceives in the environment for further processing and response. For example the responsiveness to environmental changes of patients under the conditions of an acute exacerbation or 10 years later in a more residual state are very different. In this report we take a measure of selective attention (conditioned blocking) and investigate to what extent the attention-related processes depend on sub-group diagnosis, symptom expression, age-of-onset and duration of illness.

Conditioned blocking (CB) refers to the delay in learning about the consequences of a stimulus-component ( $B$ in $A B$ ) when these consequences are already becoming associated with another component ( $A$ in $A B$ ). In other words, conditioning to the one component is said to be "blocked" by conditioning to the other (Kamin, 1969). This "blocking" is evident in healthy subjects in the delayed response to component $B$ (with respect to that to $A$ ) when the components are presented separately in the test. This "blocking" is normal because as the learning criterion of response to $A B$ is approached, responding becomes more automatic and less subject to conscious control. With the presentation of a single component when testing for the learning that has occurred for each component, information processing must be switched back to a controlled conscious mode, as was evident at the start of conditioning.

In detail, this report has four aims. First we attempted to replicate the earlier report that $C B$ is impaired only in a subgroup of patients with nonparanoid schizophrenia (Oades et al., 1996b). Second, a more precise relationship between the type of symptoms expressed and the degree of $C B$ shown was sought. This analysis was designed from the start to enable us to relate performance to the symptom clusters highlighted by Liddle (1987: psychomotor poverty, disorganization and reality distortion) and the Schneiderian first rank symptoms of ego-disturbance or ideas-of reference studied by Frith (Jahanshahi \& Frith, 1999). Third, the influence of the age of onset and duration of illness were examined. This is because the impairment of performance of patients with schizophrenia on the related task of latent inhibition (LI) is reported to dissipate with illness-duration in the longterm and medication in the shorter term (Gray et al., 1995). (LI is the delay in learning an association between a stimulus and a consequence as a result of pre-exposure to the stimulus without consequence.)

The final aim was to see if a distinction should be drawn between these associations of $\mathrm{CB}$ and $\mathrm{LI}$. To date the evidence on this issue is equivocal. It should be evident from the description of the two tasks that one would expect the mediating mechanisms to be similar if not the same. Indeed their similarity has, in the past, been argued by theorists (cf. Mackintosh, 1975). Neurobiological studies of the components necessary for $\mathrm{LI}$ and $\mathrm{CB}$ performance in animals at first showed they were similar. LI and $C B$ depend on an intact hippocampus and can be modulated by mesolimbic noradrenergic and dopaminergic activity (Solomon, 1977; Rickert et al., 1978; Lorden et al., 1980; Oades et al., 1987; Caza, 1994). However, more recently reports have appeared suggesting differences in the biological substrates. There is evidence for a stronger contribution of the frontal cortices and their monoaminergic input systems for $\mathrm{CB}$ (Oades et al., 1987) than LI (Ellenbroek et al., 1996; Lacroix et al., 1998).

Studies of patients with schizophrenia performing $C B$ also provide conflicting evidence on the similarities of the associations of $\mathrm{LI}$ and $\mathrm{CB}$. Two groups have reported on $C B$ measures in healthy subjects 
and those with schizotypal features or schizophrenia. The one has used several forms of the task successively suggesting that the chronically ill, the acutely ill or those with paranoid schizophrenia have modest problems with CB (Jones et al., 1990, 1992, 1997). Using a separate game form of $C B$ performed with a joystick we have described the development of $\mathrm{CB}$ from child- to adulthood along with its associations with dopamine and noradrenaline activity (Oades et al., 1996a). These authors also described CB in psychotic patients with paranoid and hallucinatory symptoms, but a disruption of $C B$ in patients without these positive symptoms. Increases of dopamine activity in these patients correlated with $\mathrm{CB}$, while relative increases of noradrenaline and serotonin activity interfered with CB (Oades et
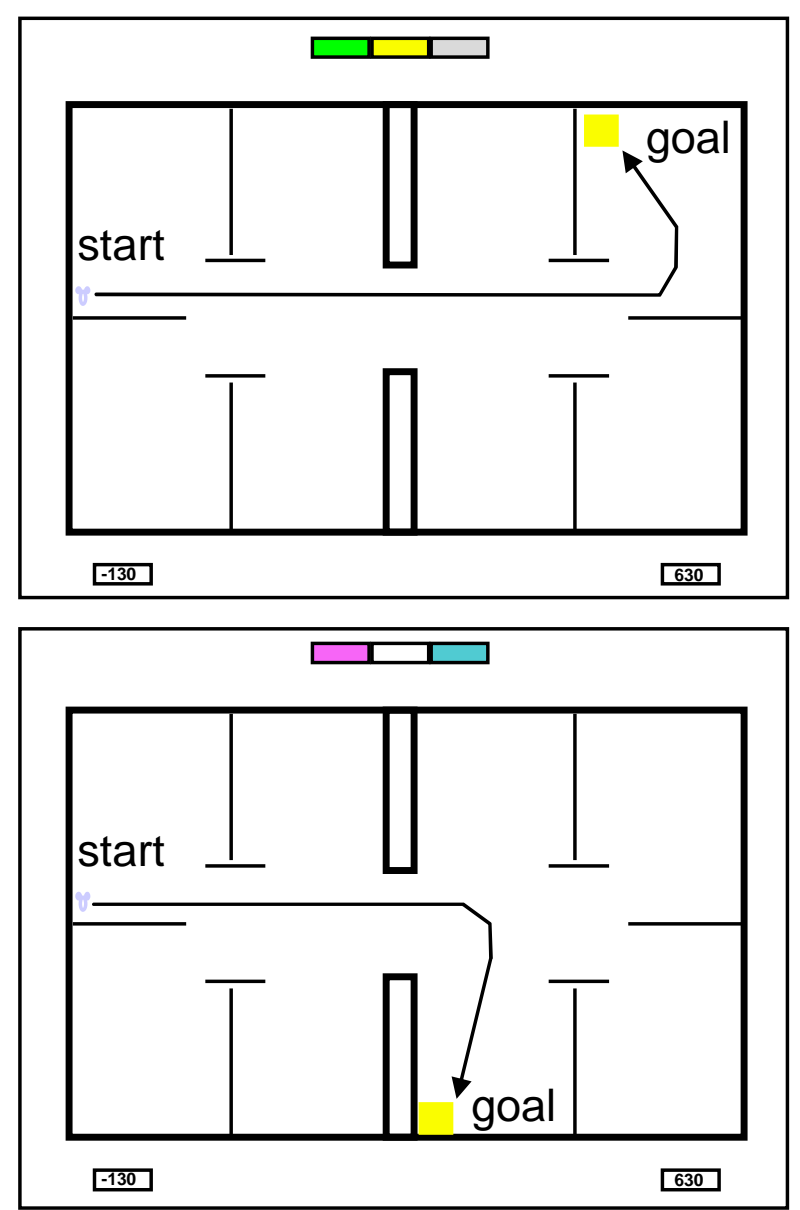

al., 1992, 1996b). The reported lack of CB disturbances in normal subjects treated with amphetamine is consistent (Gray et al., 1997).

In part 1 (section $3.1-3.5$ ), we report on a replication of the study of Oades et al. (1996b) with a new and larger group of patients, and an improved experimental design. Part 2 (section 3.6 - 3.7) describes a cross-sectional study including young and old, acute and chronically ill to see if CB depends on chronological age, duration of illness, and the age of onset. If $C B$ and $L I$ were bases on similar biological processes one would expect $C B$ to be more evident in patients who have been ill for longer.
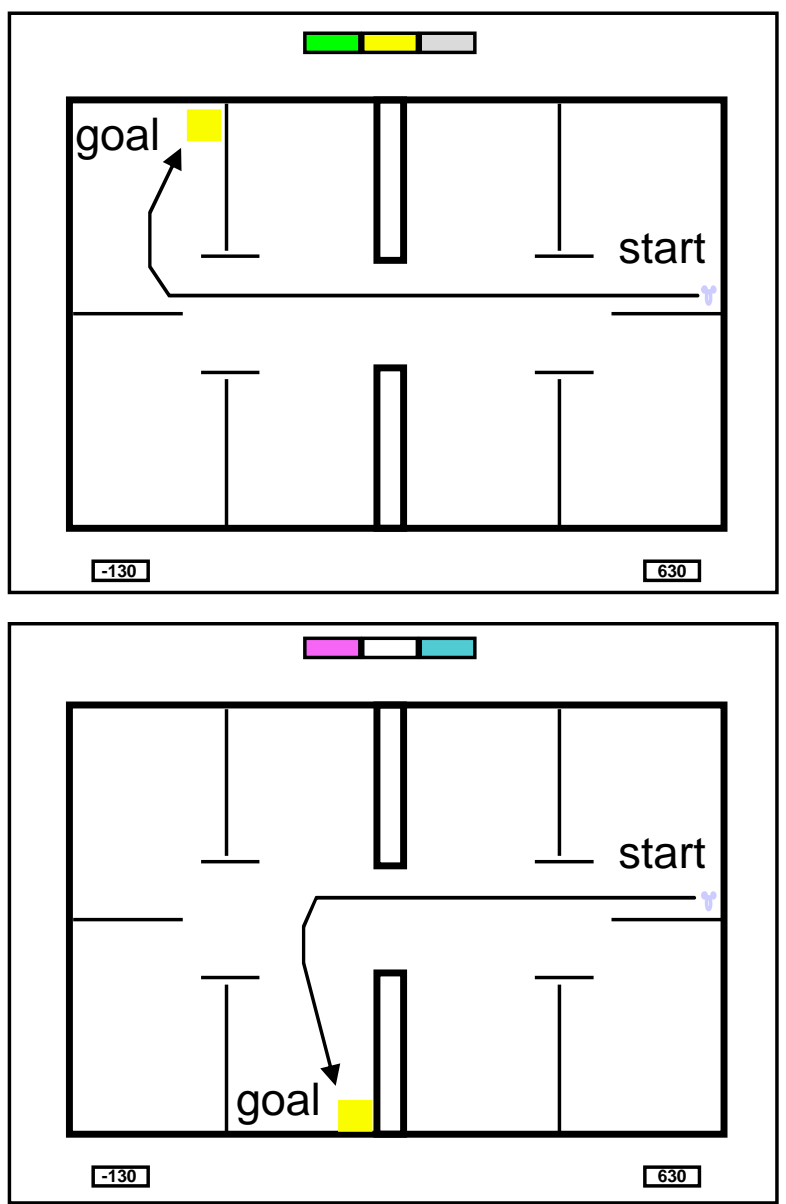

Figure 1:

Schematic drawing of 4 examples of the task situation for measuring CB. The mouse cursor appears on the left in the two left-hand examples and on the right in the right-hand examples. Three color panels (conditioned stimuli) are shown in the upper middle of each example and the potential direct route to the goal locus illustrated with an arrow. Indicators showing the plus and minus points awarded are shown below the floor-plan. 


\begin{tabular}{|c|c|c|c|c|c|c|}
\hline \multirow[b]{2}{*}{ Age (years) } & \multicolumn{3}{|c|}{$\begin{array}{l}\text { Patients } \\
\text { (+ learn criterion) } \\
\quad \mathrm{n}=62 \\
\text { Mean (S.D.) }\end{array}$} & \multicolumn{2}{|c|}{$\begin{array}{l}\text { Patients } \\
\text { (Not learn) } \\
\mathrm{n}=39 \\
\text { Mean (S.D.) }\end{array}$} & $\begin{array}{l}\text { Controls } \\
\mathrm{n}=62 \\
\text { Mean (S.D.) }\end{array}$ \\
\hline & 30.4 & (9.6) & & $37.1^{*}$ & $(12.0)$ & $32.5 \quad(11.0)$ \\
\hline Gender (m/f) & $45 / 17$ & & & $21 / 18$ & & 33 / 29 \\
\hline Socioeconomic group ${ }^{1}$ & 4.6 & $(2.0)$ & & 4.4 & $(2.0)$ & $4.9 \quad(1.6)$ \\
\hline Education (years) & 13.6 & (3.9) & & 12.6 & $(3.3)$ & $13.8 \quad(3.0)$ \\
\hline IQ (short SPM) & 7.9 & (2.7) & & $5.6^{* *}$ & $(2.6)$ & $9.9^{* *}(1.9)$ \\
\hline Hand (Edinburgh) ${ }^{2}$ & 16.8 & (9.6) & & 18.7 & $(6.0)$ & $18.9 \quad(5.3)$ \\
\hline \multicolumn{7}{|l|}{ Diagnosis (DSM-IV) } \\
\hline Disorganized & 16 & & & 8 & & \\
\hline Catatonic & 2 & & & 1 & & \\
\hline Paranoid & 41 & & & 29 & & \\
\hline Residual & 3 & & & 1 & & \\
\hline Onset-age (years) & 23.2 & $(8.1)$ & & 23.3 & $(7.3)$ & \\
\hline Illness-duration (years) & 7.2 & (6.4) & & $13.4^{\#}$ & $(8.8)$ & \\
\hline Episode-duration (days) & 44.3 & $(45.0)$ & & 43.6 & $(35.6)$ & \\
\hline \multicolumn{7}{|l|}{ Symptoms } \\
\hline \multicolumn{7}{|l|}{ PANSS sum (scale 1-7) } \\
\hline Positive ( 7 items) & 15.5 & $(5.9)$ & & 17.6 & $(6.2)$ & \\
\hline Negative ( 7 items) & 18.1 & $(8.4)$ & & 19.5 & $(7.8)$ & \\
\hline General (16 items) & 36.0 & $(9.7)$ & & 38.1 & $(9.1)$ & \\
\hline \multicolumn{7}{|l|}{ SAPS (scale 0-5) } \\
\hline Ideas/reference (5 items) & 3.0 & $(3.3)$ & & 3.5 & $(4.5)$ & \\
\hline Thought-disorder ( 7 items) & s) 8.2 & $(7.3)$ & & 8.8 & $(5.6)$ & \\
\hline EPS & 3.0 & $(4.1)$ & & 4.8 & $(6.7)$ & \\
\hline AIMS & 7.9 & $(2.4)$ & & 8.2 & $(2.9)$ & \\
\hline \multicolumn{7}{|l|}{ Medication } \\
\hline Neuroleptic & 617 & $(340)$ & $(\mathrm{n}=59)^{\mathbf{a}}$ & 732 & $(311)(n=39)$ & (Chlorpromazine \\
\hline 'typical' & 513 & $(351)$ & $(n=32)$ & 698 & $(297)(n=15)$ & equivalents) \\
\hline Clozapine/olanzapine & 742 & $(283)$ & $(\mathrm{n}=19)$ & 694 & $(311)(n=18)$ & \\
\hline Both & 740 & $(306)$ & $(\mathrm{n}=8)$ & 928 & $(319)(n=6)$ & \\
\hline Biperidene (mg) & 4.2 & $(1.6)$ & $(n=9)$ & 4.8 & $(1.8)(n=5)$ & \\
\hline
\end{tabular}

1: Scale 1-7; Brauns et al., 1997 2: -24 (left) to + 24 (right-handed).

* age $\mathrm{p}<0.003(\mathrm{t}=-3.1$, between patient groups); $* * \mathbf{I Q} \mathrm{p}<0.001(\mathrm{t}=+4.3$, between patient groups, $\mathrm{t}=-4.6$ between subjects learning CB), \# illness-duration $\mathrm{p}<0.001(\mathrm{t}=-4.0$, between patient groups), a: 3 patients were medication-free

\section{Methods}

\subsection{Subjects}

From 101 patients with schizophrenia presented with the conditioned blocking (CB) task, 62 achieved a degree of initial learning permitting a comparison with the performance of 62 healthy subjects (criterion $7 / 10$ trials correct in under $10 \mathrm{sec}$ ), group- 
matched for age, years spent in education and socio-economic background (see Table 1 and below).

Informed and signed consent about the purposes of the study was obtained from each of the 90 inpatients, 11 outpatients, their responsible care-givers and the healthy controls. Patients were recruited from the child, adolescent and adult psychiatry clinics and initial diagnosis was confirmed by the senior ward physician. These patients were reexamined for entry to the study by two senior physicians of the research group (DSM-IV, criteria A-E, American Psychiatric Association, 1994). Thus affective, schizoaffective and schizophreniform psychoses were excluded. Patients were additionally screened to exclude other major psychiatric or somatic illness, alcohol abuse in the last 5 years, and substance abuse other than nicotine. Schizophrenia subtypes were also defined by DSM-IV criteria, whereby the undifferentiated type was regarded as a residual category that contrasts with the paranoid, disorganized and catatonic subtypes, (for clinical assessments and medication, see Table 1).

Symptom severity was rated according to the Positive and Negative Syndrome Scale (PANSS: Kay et al., 1992) and, as ideas-ofreference and thought disorder are under represented in this rating scale, the relevant items 15-19 and 26-32 from the Scale for Assessment of Positive Symptoms (SAPS: Andreasen, 1984) were also scored. Clinical ratings included 3 scales rating extrapyramidal symptoms (Simpson-Angus Scale, 1970), Abnormal Involuntary Movement Scale, AIMS, and the Barnes Akathisia Scale (1989). Handedness was evaluated with the Edinburgh inventory (Oldfield, 1971).

Illness-onset was defined as the age at onset of psychosis as assessed by the therapist on interview with the patient and usually from information from a relative: for some older patients this had to be set as the date of first admission (range 8.9-45.8y). Illness-duration was taken as the time elapsing between illness-onset and testing (range 0.02-20.1y). Among inpatients, the duration of the current admission to the clinic ranged from 1 to 211 days. Three patients were without medication at the time of the test, 40 received atypical (clozapine and olanzapine) and 40 other more typical neuroleptic medications (including risperidone: Table 1). Fourteen patients received biperidene (mean $4.4 \mathrm{mg}$, range 2-8 $\mathrm{mg}$ ).

The 62 healthy control patients were recruited by advertisement and were paid for participation. The selection controlled for the socio-economic distribution and age among the patients: gender ratios were not significantly different (Table 1). The exclusion criteria, based on a semi-structured interview were the same as described for the patients. In addition they reported no family history of psychotic illness, nor that they had previously consulted with a psychiatrist or psychologist.

\subsection{Testing procedures}

The $C B$ task was introduced as a computer game. A mouse-like cursor could be moved with a joystick through a maze resembling the floor-plan of an apartment (see Fig. 1: white plan on black background). Subjects started in pseudo-random order from the left or right sides of the maze and were asked to find a goal in the other room with the cursor. On reaching the goal the locus would shimmer yellow and 30 points were awarded on counters below the floor-plan. Every second beyond a latency of $8 \mathrm{sec}$ was scored with minus one point per second. Such trials were scored as 'errors' for the calculation of the learning criterion. The inter-trial-interval was 2 seconds. The reaction time achieved by skilled subjects was about $2.4 \mathrm{sec}$.

In practice, to achieve a reasonable information load there were not one but two discrimination tasks with different goals. The cue for starting a trial and for association of the correct goal locus consisted of a series of color-panels located above the floor-plan for $2 \mathrm{sec}$ at the start of each trial (Fig. 1). The two discrimination tasks were presented in a pseudo-random sequence. All learning sessions ended with a phase during which 3 color-panels were presented: there followed two trials with only the single panel from the middle of the panel-array. The data for these 
two trials were not analyzed: their presence controlled for and limited the effect of surprise in the presentation of one rather than three color-panels. (Oades et al., 1996b reported that the first single-panel test-trial latencies were long and had to be discarded.) Test trials followed with single presentations of the colors that had appeared on the left or the right of the panel arrays during learning.

$C B$ requires that during learning a stimulus is added (e.g. $B$ to $A$ ). Thus on such a blocking session two color-panels $(=A)$ were presented up to a learning criterion of $5 / 8$ correct responses, when a third color-panel (B) was added up to full acquisition (7/8 correct). But blocking performance can only be judged by reference to response latencies acquired when all 3 panels were present from the beginning. In this report the reference session was presented on the next day after the blocking session as a procedural improvement on our previous report.

What was the measure of $C B$ used? Single color-panels were presented in sequence for 12 test trials. Subtraction of the latency of response to the added color-panel from that for the other present from the start of learning gave the within-session blocking score. Subtraction of the similar value obtained on the reference session (right panel minus left panel latency) from those on the blocking session gave the actual CB scores used. Thus the "first trial" test measure represents a double subtraction procedure (from 4 responses on 2 sessions) for one discrimination: the first "trial-pair" measure represents the average of the doublesubtraction procedures for the first test of each of the two discriminations.

Here a comment is appropriate about the 3 methodological changes under-taken since the original report (Oades et al., 1996b). Pilot work suggested that all 3 changes would be improvements. During the study it became evident that two of these changes were responsible for extending the learning phase and frustrating patients to an extent that criteria for CB could not be met in 39/101 patients. (The control subjects were not so affected.) These were the inclusion of a T- hook on the end of the walls of the maze to slow skilled dashing to all corners of the maze, requiring no learning, and the confusion derived from the middle-panel presentations introduced to control for a 'surprise effect'. The reversed order of presenting the blocking and reference sessions is a procedural improvement on the previous report.

\subsection{Data analysis}

Demographic and clinical data were compared with Student's t-test, and Kendall tau correlation coefficients are reported (Arndt et al., 1999). CB data, with separate treatment for initial $C B$ (double-difference on the first trial-pair, $X 12$ ) and late $C B$ (trial-pair X56) were analyzed by MANOVA, with repeated measures where the data came from several trials, with age as a covariate. (The distribution CB data X12 and X56 did not differ significantly from normal, $\left(\chi^{2}=10.1\right.$ to 4.6, $\mathrm{p}=0.12$ to 0.59, CSS: Statistica, 1991). The contribution of symptoms rated and aspects of the age of onset and illnessduration to $C B$ measures were investigated with the use of linear regression models. The examination of the major symptom clusters (and contributory symptoms) was supported by a principal component analysis of the PANSS psychotic symptoms, and SAPS ideasof-reference and thought-disorder items. Selection of factors for varimax rotation was based on Eigen values larger than one. Exclusion of dimensions contributing less than $5 \%$ to the variance resulted in 4 dimensions explaining $60 \%$ of the total variance (i.e. 1 . SAPS thought disorder + PANSS conceptdisorder, 2. 6/7 PANSS negative symptoms, 3. SAPS ideas-of-reference, 4. PANSS positive symptoms).

\section{Results}

3.1 (Part 1) Demography: group comparisons

Patients and controls who learned the task were matched for age, socio-economic status, and time in education. The patient group as a whole, but particularly those who did not reach a satisfactory learning criterion scored less than the controls on the short APM-IQ test (Table 1). Although the patients who did not complete the task were on average 6.7 years older and had been ill for 6.2 years longer than those who did learn, the two 
patient groups did not differ on symptom ratings, level of medication, and age of onset.

\subsection{Relations of demography to learning, and $C B$}

Faster learning (fewer trials) was associated with increased IQ in controls but not in patients $(\tau=-0.26, p=0.003)$ : however there was no association with the time spent in education. Among controls higher parental socio-economic status related to faster learning ( $\tau=+0.31, p=0.0006)$; this feature showed as a trend for patients $(\tau=0.16, p=$ 0.08 ). In contrast increasing age in patients was more obviously associated with slower learning than in controls ( $\tau=+0.26$ vs. $+0.16, p$ $=0.004$ vs. 0.06 ) .

Blocking ( $C B$ X12) was related to age and gender, but not to other features like handedness or IQ. CB correlated strongly with age in controls, but not in patients $(\tau=+0.29$, $p=0.0008$ ). Thus, age was used as a covariate in further group comparisons. Gender comparisons showed that male controls learned with fewer trials than females $(\mathrm{t}=$ 2.2, $p=0.03)$. Gender did not influence the degree of initial CB (X12) shown in patients or controls: but at the end of testing $C B$ scores (X56) remained high in female patients $(6.69$ vs. $1.99, \mathrm{t}=-2.7, p=0.008$ ), while in male patients scores decreased reflecting normal learning about the single cue presentations. (A detailed analysis of gender effects is in preparation)

\subsection{CB in patients with schizophrenia and in paranoid vs. nonparanoid diagnostic subgroups}

The controls showed the normal pattern of $C B$ on the first trial-pair that was nearly halved on the second, and halved again on the third trial-pair (Fig. 2 left). This reflects learning about the single stimuli - tending to respond to each with a similar latency as the testphase proceeds.

A main group difference for $C B$ scores across the first 3 trial-pairs was shown by a repeated measure MANCOVA $(F[1,118]=$ 9.89, $p=0.002$; Fig. 2). $C B$ in controls decreased, while in patients $C B$ persisted or increased slightly across test-trials, resulting in higher scores than controls by the third trialpair $(F[1,118]=10.18, p<0.002)$.

What types of patients showed high and low CB scores? As Oades et al. (1996) reported impaired $C B$ in patients with negligible paranoid and hallucinatory symptoms, initial CB was compared between those with a diagnosis of paranoid schizophrenia $(n=42)$ and those with other nonparanoid diagnoses $(n=20)$. The paranoid group showed good CB (3.3 sem $1.2 \mathrm{sec}$ ) while the nonparanoid group showed an absence of CB $(-1.1$ sem 1.6 sec: $F[1,58]=4.78, p=0.03$; Fig. 3). Restriction of the analysis to patients under 26 years of age (mean 18.3y), as in the previous report, yielded 12 with and 6 without a diagnosis of paranoid schizophrenia. The difference in $C B$ was not significant in this small sample, but it reflected the earlier report with low CB scores in the nonparanoid group (2.2 vs. $1.2 \mathrm{sec}$ ).

\subsection{CB as a function of clusters of symptoms expressed}

Our previous analysis, based exclusively on SANS and SAPS ratings, could not be exactly replicated as symptoms were rated on the PANSS, along with SAPS ratings of ideas-ofreference and thought disorder. Factor analysis of the symptoms rated by PANSS and the two SAPS clusters resulted in four meaningful dimensions. However, none of these symptom groupings gave a significant model in a linear regression analysis of $C B$ (X12). But a significant trend was obtained for the 5 sum scores from the rating scales (positive, negative, general symptoms from PANSS, ideas-of-reference and thought disorder from SAPS) that broad-ly reflected the factor-analysis $(\mathrm{F}[5,55]=2.15, p=0.07)$. A stepwise regression showed that after removal of the three PANSS clusters $(F[2,58]$ $=5.02, p<0.01$ ), a significant positive contribution was made by thought disorder ( $B$ $=0.35, p=0.008$ ) and a negative contribution by ideas-of-reference explaining $15 \%$ of the variance $(B=-0.29, p=0.02$, see partial correlations in Fig. 4a). Reduced CB in patients expressing mild thought disorder or severe ideas-of-reference vs. increased $C B$ in those with severer thought disorder and mild ideas- 
of-reference was demonstrated by an analysis of variance covarying for age, IQ

\section{Figure 2 (below)}

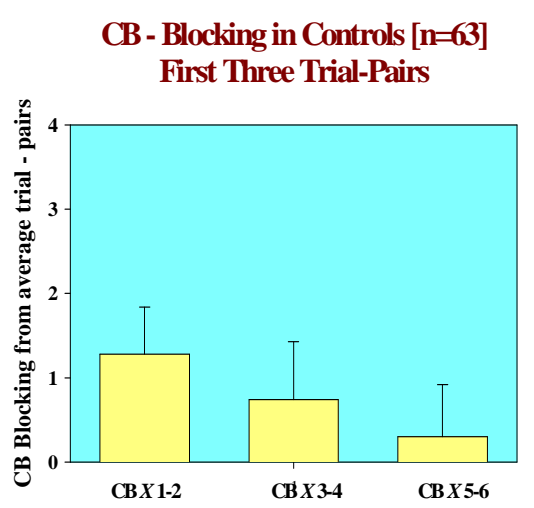

CB - Blocking in SCH Patients [n=61] First Three Trial-Pairs

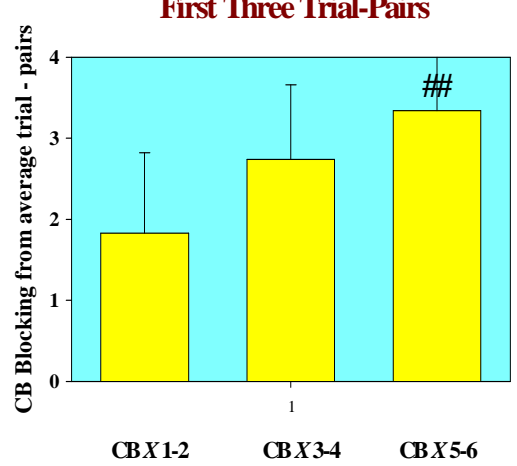

\section{Figure 3 (below)}
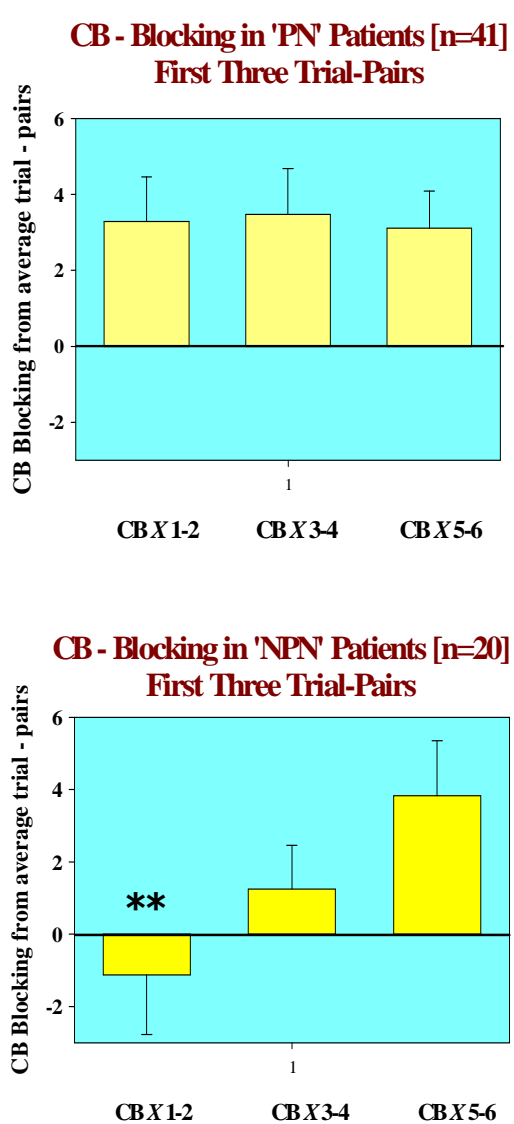

\section{Figure 2:}

Above - are shown the derived CB latency differences (sec) for 62 control subjects, for the first, second and third trial-pair presentations: (i.e. mean response latency to the originally right-hand stimulus-panel, less that to the originally left-hand panel in the blocking minus the reference session for the 2 discriminations, and sem) The decrease of $\mathrm{CB}$ with exposure to the single conditioned stimulus components in the test is illustrated. Below - the same CB measures are shown for 62 patients with schizophrenia, illustrating the persistence of $\mathrm{CB}$ with test trials. \#\# $p<0.002$ (within and between groups, see text).

\section{Figure 3:}

Above - the persistence of $\mathrm{CB}$ (sec, sem) in patients with paranoid schizophrenia (PN) is contrasted below with the absence of initial CB in patients without a nonparanoid diagnosis (NPN). $* * p=0.03$ (group comparison).

and neuroleptic medication $(F[1,55]=3.97$, $p=0.05)$ and is shown in the insert of figure 4 .

The conclusions of this analysis were confirmed by a stepwise regression of the PANSS symptoms, alone. The last symptoms in the solution were concept disorder $(B=$ $+0.44, p<0.0004)$ and persecutory delusions $(B=-0.33, p=0.007$ ) which are the PANSS ratings related to and factoring with thought disorder and ideas-of-reference $(F[5,53]=$ $6.5, p<0.0004)$. Prior to this step significant negative contributions were also made by poor-rapport, poor-attention and a positive contribution by apathy $(\mathrm{F}[9,49]=4.79, p=$ $0.00014)$. This example is illustrated in figure $4 \mathrm{~b}$ for comparison.

A regression model for late $C B$ test trials (third trial-pair) did not reach conventional 
levels of significance, but a consideration of the PANSS positive and negative symptoms illustrates the trend $(F[14,35]=1.67, p=$ $0.10)$. In this model there was a weak positive association for continued high CB scores with concept disorder, a positive symptom, and a marked negative association pointing to low CB scores associated with flat-affect, a negative symptom.

\subsection{Effects of Medication}

There was no correlation between CB scores and neuroleptic doses calculated as chlorpromazine equivalents. Nine patients receiving the anticholinergic biperidene hydrochloride performed the CB task. Surprisingly with this small sample and a dose range restricted to $8 \mathrm{mg}$ (mean $4.2 \mathrm{mg} \mathrm{SD}$ 1.5), treatment tended to correlate with $C B(\tau$ $=0.43, p=0.086)$. Indeed CB for the treated group was double the average for the remaining patients $(5.7 \mathrm{sem} 2.6$ vs. $2.3 \mathrm{sem}$ 1.1). This point was further explored by studying those receiving the atypical agents clozapine or olanzapine with muscarinic binding properties and other neuroleptics with a more typical action on catecholamine binding sites. This indicated a closer, albeit non-significant relationship for treatment with typical neuroleptics with CB scores: (typical neuroleptics, mean 513 S.D. 351 equivalents, $\tau=+0.17, p=0.18, \mathrm{n}=32$ vs. atypical neuroleptics, mean 739 S.D. 281 equivalents; $\tau=-0.13, p=0.41, \mathrm{n}=20$ ). In the absence of a significant dose difference, this points more to a modest role for catecholamines rather than for acetylcholine in the mediation of CB.

\subsection{Summary (Part 1)}

$C B$ scores were higher in this sample of patients with schizophrenia than reported by Oades et al. (1996b). This is attributable to the high proportion of those with a diagnosis of paranoid schizophrenia. The present study replicates the earlier report by finding $C B$ expressed by those with paranoid schizophrenia and impaired in patients with nonparanoid schizophrenia. Indeed CB performance was positively associated with productive symptoms, including concept disorder. Similarly, we confirm that low $C B$ scores were associated with some negative symptoms (e.g. poor rapport and attention). The novel difference reported here is that low CB scores were also associated with ideas-ofreference. An additional new finding was that high CB scores persisted more in female patients and those with high thought disorder ratings. Conflicting evidence is presented on a potential contribution of anticholinergic medication.

\section{7 (Part 2) Illness-onset and illness-duration}

There were no significant differences in onset-age, illness-duration and current episode duration between the patients that did and those that did not acquire the task. None of these characteristics of the illness correlated with CB (first trial-pair) nor did they differ significantly between the paranoid patients who showed $C B$ and the nonparanoid patients that did not (paranoid/nonparanoid: onset at 26.6 vs. $23.3 y$, duration 6.4 vs. $5.3 y$, episode 45.8 days vs. 58.2 days). Nonetheless a slightly earlier onset and longer stay on the wards in those showing less CB suggested an examination of their contribution to the variance in a regression.

Chronological age, onset-age, illnessduration and episode duration were entered into a backward stepwise regression for initial $C B$. Removal of the trivial contribution of episode-duration ( $\beta=-0.1, p=0.4$ ) resulted in a model of trend significance $(F[3,44]=2.28$, $p=0.09$ ). In the light of the significant weights this indicated that $C B$ tends to be present in younger patients (age, $\beta=-15.6$ ), but in those with a later age of onset $(B=+15.6)$ and longer duration $(B=+10.1)$. This model improved if only those with a diagnosis of paranoid schizophrenia were considered $(F[4$, $27]=2.4, p=0.07$ ), but was not significant for the small number of patients with a nonparanoid diagnosis, nor when applied to later CB test-measures (X56).

\subsection{Summary (Part 2)}

There was a weak trend for less CB to be shown in the older among the patients who showed an earlier than average age of onset of the illness. Features describing the length of the illness (episode or diagnosis) were not related to $\mathrm{CB}$ reduction. 

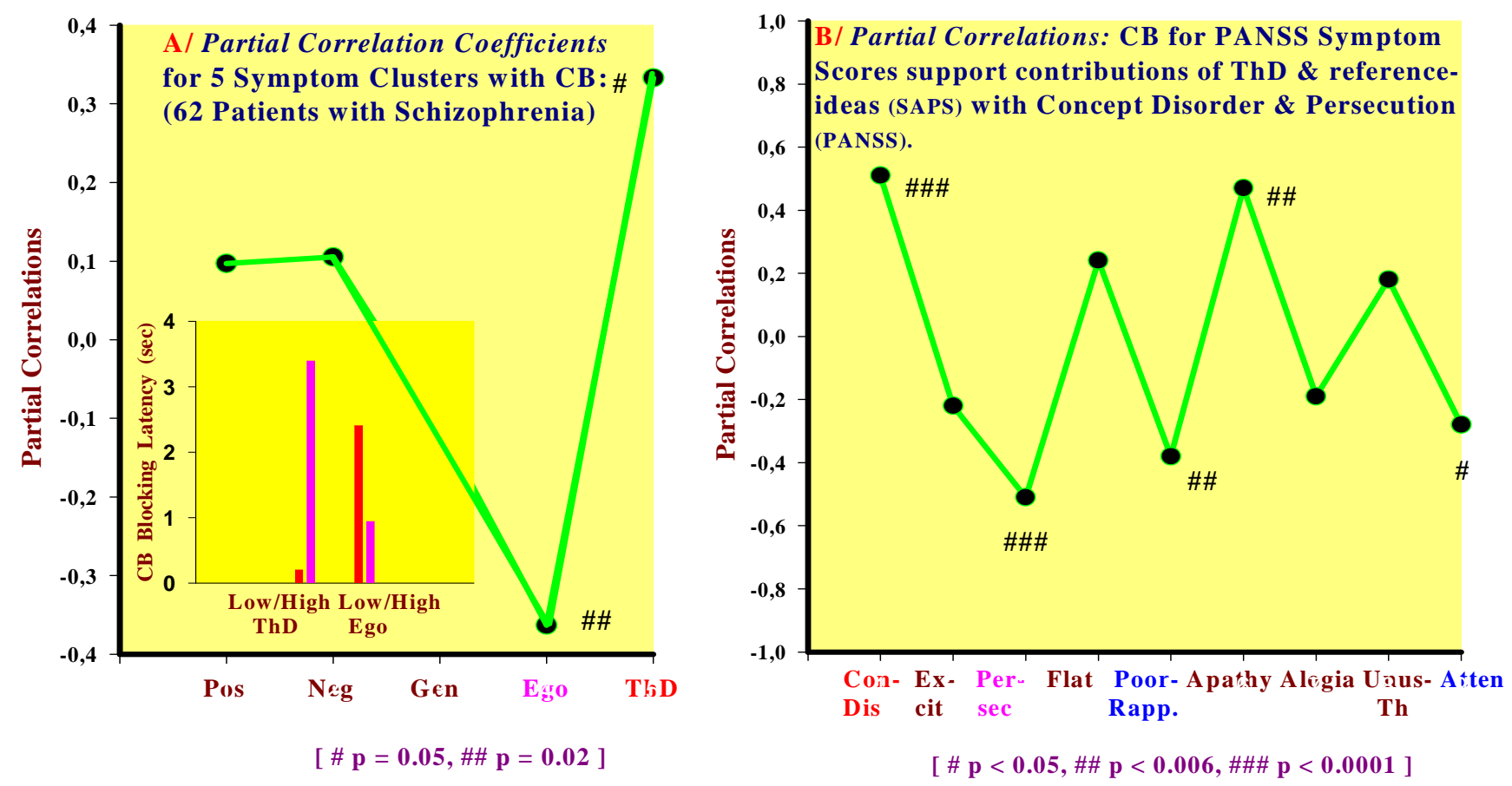

\section{Figure 4:}

On the left: The profile of partial correlation coefficients for positive (Pos), negative (Neg), general (Gen) symptom-clusters (PANSS), with ideas-of-reference (Ego) and thought disorder symptom groupings (ThD: SAPS). The latter two contribute negatively and positively, respectively, to the expression of initial CB (\# $\mathrm{p}=0.05$, \#\# $\mathrm{p}=0.02)$. The insert shows reduced $\mathrm{CB}$ in patients expressing little thought disorder (ThD) or much ego/ideas-of-reference disturbance (see text). On the right: a representative regression step for PANSS psychotic symptoms illustrates the similar major positive and negative contributions respectively, from Concept-Disorder (Con-Dis) vs. Persecution (Persec) and Poor-Rapport (Poor-Rapp.) \# p $<0.05$, \#\# p < 0.006, \#\#\# $<$ 0.0001). Contributions from excitation (Excit), flat-affect (Flat), Alogia and unusual thoughts (Unus-Th) were not significant.

\section{Discussion}

\subsection{Main Effects}

There were five main results. First, conditioned blocking (CB) was not impaired in a large cross-sectional sample of patients with schizophrenia, taken as a whole. Second, CB was clearly reduced in patients with nonparanoid diagnoses of schizophrenia (disorganized, catatonic or residual subgroups). Third, the CB shown by patients with a diagnosis of paranoid schizophrenia persisted over more test-trials. Fourth $C B$ expression was associated with positive thought disorder-related symptoms, and $C B$ reduction related to ideas-of-references and negative symptoms such as poor rapport and poor attention.
Thus, our first main expectation was fulfilled: namely this report replicates the finding of Oades et al. (1996b) that patients with diagnoses of nonparanoid schizophrenia showed impaired CB. This amply supports the call from Carpenter and colleagues (1999) to research workers to distinguish between the patients that do and those that do not contribute to the effects measured. The important novel finding is that classic delusions (e.g. of persecution) should be grouped with hallucinations in regard to their associations with $\mathrm{CB}$ expression, while the Schneiderian first rank symptoms of ideas-ofreference are associated with less CB.

Fifth and last, the present results contrast with expectations based on LI studies, namely that an impairment in acutely ill patients 
should dissipate with time or medication. Impaired CB performance was independent of illness duration and neuroleptic dose. The results are consistent with much of the neuropsychological literature showing that patients with a younger onset-age are more likely to show an impaired cognitive performance (e.g., Trails and Card-sorting: abstraction and flexibility) and to manifest negative symptoms (Hoff et al., 1996; Jeste et al., 1998).

\subsection{Methods}

A brief consideration of the changes in the task presentation with respect to our previous report is appropriate. These may be summarized as concerning, a) balance (the blocking session was presented before the reference session), b) structure (edges were added to the sides of the maze to reduce fast random dashing about the maze), c) the program (the addition of two trials after learning and before test to reduce the effect of surprise of single cue panels that delay initial response latencies). With regard to 'balance, it is encouraging that the CB effect does not depend on the sequence of presenting the reference (learning-control) and $C B$ sessions. The presence of $C B$ or its impairment in some patients was evident independent of the order of presentation. In contrast, increasing the search difficulty with extensions of the sides to the floor-plan for the task was mistaken. This compounded the problems facing patients on a task that already and necessarily had a high information load (two simultaneous discriminations). It probably accounted for the increased age for the group of patients that could not learn the computer-based joystick task satisfactorily. The absence of correlations with extrapyramidal symptoms suggests that the matter was not simply one of being physically disadvantaged. Lastly, the program change instituted two single-panel pre-test trials after the learning criterion had been achieved to dissociate surprise effects from $\mathrm{CB}$ test-trials. While the change was successful in removing the surprise effect, it unwittingly reinforced the tendency to show $C B$, as the panels had also been present from the beginning of learning.

\subsection{Clinical associations of normal/abnormal $C B$}

A distinction between the cognitive styles of paranoid and nonparanoid patients with schizophrenia has been recognized for a long time (Kahlbaum, 1863; Magaro, 1980). According to these authors nonparanoid patients rely more on perceptual and less on contextual aspects of a situation to solve a task. This feature is amply shown by the nonparanoid patients in the present report being able to learn about both the original and the added stimulus components of the $C B$ task: conscious controlled processing occurs from the start to the end of the task. In contrast paranoid patients are better able to switch in an active controlled processing strategy (Heim, 1989), and hence here to show 'normal' CB. The unexpected implication from the present results is that persistent automatic processing may be associated with states where little thought disorder but many ideas-of-reference are expressed. Let us consider the contribution of these two symptom clusters.

Thought and concept disorder are considered to be positive symptoms (Andreasen, 1984; Kay et al., 1992). Their absence parallels the association of some negative symptoms with impaired $\mathrm{CB}$. The presence of schizophrenic thought disorder is prominent among symptom clusters associated with patients' difficulties with verbal memory, abstraction and executive function (Nestor et al., 1998). From the literature it is less apparent with what the absence of thought disorder is associated. However, a plausible explanation may lie in drawing a parallel with semantic priming. This priming effect refers to the facilitated response latency to a second stimulus, where its features are related to those of the first stimulus presented. The priming effect is larger in schizophrenic patients with than those without thought disorder (Weisbrod et al., 1998). We suggest that the initial conditioned stimulus in the $C B$ task takes the role of a priming stimulus. It exerts more control and a more persistent control over responsiveness to associated stimulus components that leads to normal $\mathrm{CB}$ and the 
continued expression of CB. Low levels of thought disorder would be permissive of other influences that lead to $C B$ absence. Nonparanoid patients (expressing little positive thought disorder) are those whom Magaro (1980) suggests do not use the context of the situation in associative learning, retain controlled perceptual processing strategies, and thus here would not manifest a CB effect. But how does the contribution of marked ideas-of reference to $\mathrm{CB}$ impairment fit into this viewpoint?

Patients with ideas-of-reference and thoughts of alien control have been attributed with an impaired ability to monitor information processed in the frontal and temporal lobes (Jahanshahi \& Frith, 1999). Stirling et al., (1998) confirmed their hypothesis that patients exhibiting such ideasof-reference would have especial problems on neuropsychological tests requiring selfmonitoring, such as visual reproduction. We have also found evidence in support of an interpretation in terms of impaired monitoring. In the current study perseverative verbal fluency errors were an especial feature of patients with marked ideas-of reference, and a significant proportion of the variance of CB was accounted for by visual reproduction performance (Oades et al., 2000). Furthermore, Gray (1998) viewed the disruption of monitoring described by Frith as the main feature manifested by the symptoms of ideas-of-reference: in turn he interpreted this as a disruption of 'efferent copy' from frontal to hippocampal regions. These points underline the importance of viewing the symptom cluster 'ideas-of-reference' as a separate entity, and are consistent with the general implications from animal studies of limbic-frontal connectivity being significant for the functions necessary for $\mathrm{LI}$ and $\mathrm{CB}$ performance. However, we must briefly consider the implications of our results on illness duration for the differences between $\mathrm{LI}$ and $C B$ results.

4.4 Influence of onset-age and illnessduration, the state-trait features

The question of whether the cognitive problems of patients with schizophrenia reflect the psychotic state or persist as a trait independent of the overt symptoms is important for understanding the bases for the expression of anomalous information processing as well as for the accessibility of the symptoms to therapeutic measures. Features of sustained attention have often been claimed to reflect a trait associated with the diagnosis of schizophrenia (Addington and Addington, 1998; Finkelstein et al., 1997). LI measures of selective attention seem to be more state-dependent in that the acutely but not the chronically ill show disrupted LI (Baruch et al. 1988). The difference remains even in patients matched for their positive symptom scores (Gray et al., 1992). These authors found that LI may be disrupted in the first two weeks after admission, but could be normalized after 8-12 weeks of neuroleptic treatment. Subsequently they confirmed this effect independent of medication (Gray et al. 1995). Illness duration correlated linearly with the $\mathrm{LI}$ effect, and crossed over to normal performance after a year.

Features related to illness are broadly unrelated to $\mathrm{CB}$ expression. $\mathrm{CB}$ tended to be present in younger patients, who may be more adept at performing computer based tasks, but CB was more impaired in those with an earlier onset, reflecting tendencies reported for other cognitive abilities (Jacobsen and Rapoport, 1998), and in those with a longer period since original diagnosis. Indeed, the lack of similar sensitivities for LI and $C B$ tasks to the features of illness progression indicates a lack of similarity in the underlying substrate. On the basis of animal and human work Gray et al., (1991) elaborated a parahippocampal (subicular) mesolimbic dopaminergic substrate for LI. Somewhat similarly, on the basis of animal (Oades et al. 1987) and human work (Oades et al., 1996a, b) we proposed for $C B$ an interaction between the nodes on which the mesolimbic, but also the mesocortical dopaminergic pathways act (frontal and limbic systems). This is consistent with the discussion (above) on the monitoring of processes between the frontal and temporal lobes 
In conclusion, the presence of normal CB in patients with a diagnosis of paranoid schizophrenia and positive thought disorder is consistent with both our own previous report (Oades et al., 1996b) and that from the treatment of healthy subjects with amphetamine (Gray et al., 1997), previously described as a model for aspects of paranoid schizophrenia (Connell, 1958). We interpret the persistence of $C B$ through testing in terms of Hemsley's (1987) hypothesis as the persistent use of an automatic stimulus processing strategy. In contrast our renewed finding that reduced $C B$ is associated with the nonparanoid diagnosis along with the novel finding of an association with ideas-ofreference is interpreted as the persistent use of controlled stimulus processing strategies.

In one sense information processing strategies reflected by $C B$ performance of patients with schizophrenia are statedependent: not all such patients are impaired, and in those that are, the impairment is related to specific symptoms. On the other hand these symptoms have negative characteristics or contain ideas-of-reference of the type known to persist to a greater or lesser degree like a trait in the chronic syndrome. Indeed, there was little evidence in this cross-sectional analysis, that the older or more chronically ill were any less likely to be impaired, as would be expected from statedependent characteristics or the data from $\mathrm{LI}$ studies. A firm statement on this issue however needs to be based on longitudinal data.

\section{References}

Addington, J. and Addington, D., 1998. Visual attention and symptoms in schizophrenia: a 1-year follow-up. Schizophr. Res. 34, 95-99.

American Psychiatric Association, 1994. Diagnostic and Statistical Manual of Mental Disorders (DSM-IV), $4^{\text {th }}$ ed., American Psychiatric Association, Washington, D.C.

Andreasen, N. C., 1984. The Scale for the Assessment of Positive Symptoms (SAPS) University of lowa, lowa City, IA.

Arndt, S., Turvey, C., Andreasen, N. C., (1999). Correlating and predicting psychiatric symptom ratings: Spearman's r vs. Kendall's tau correlation. J. Psychiatr. Res.33, 97-104.

Barnes, T. R. E., 1989. A rating scale for the drug-induced akathisia. Br. J. Psychiatry 154, 672-676.

Baruch, I., Hemsley, D. R., Gray, J. A., 1988. Differential performance of acute and chronic schizophrenics in a latent inhibition task. J. Nerv. Ment. Dis. 176, 598-606.

Brauns, H., Haun, D., Steinmann, S., 1997. The construction of an internationally comparable classification by class. Erwerbsstatistische Besonderheiten am Beispiel von Labour Force Surveys der Bundesrepublik Deutschland, Frankreichs, Großbritanniens und Ungarns. Arbeitspapiere Arbeitsbereich 1/22, Mannheim Center for European Social Research (MZES), Mannheim.

Carpenter, W. T., Kirkpatrick, B., Buchanan, R. W., 1999. Schizophrenia: syndromes and diseases. J. Psychiatr. Res., 33, 473-475.

Caza, P. A., 1994. Noradrenergic influences on blocking: interactions with development. Pharmacol. Biochem. Behav. 21, 9-17.

Connell, P., 1958. Amphetamine Psychosis. Chapman \& Hall, London.

CSS: Statistica. 1991. Complete Statistical System. Statsoft, Tulsa, OK.

Ellenbroek, B. A., Budde, S., Cools, A. R., 1996. Prepulse inhibition and latent inhibition: the role of dopamine in the medial prefrontal cortex. Neurosci. 75, 535-542.

Finkelstein, J. R. J., Cannon, T. D., Gur, R. E., Gur, R. C., Moberg, P., 1997. Attentional dysfunctions in neuroleptic-naive and neuroleptic-withdrawn schizophrenic patients and their siblings. J. Abnorm. Psychology 106, 203-212.

Gray, J. A., (1998). Integrating schizophrenia. Schizophr. Bull. 24, 249-266.

Gray, J. A., Feldon, J., Rawlins, J. N. P., Hemsley, D. R., Smith, A. D., 1991. The neuropsychology of schizophrenia. Behav. Brain Sci. 14, 1-20.

Gray, N. S., Hemsley, D. R., Gray, J. A., 1992. Abolition of latent inhibition in acute but not in chronic schizophrenics. Neurol. Psychiatry Brain Res. 1, 83-89.

Gray, N. S., Pilowsky, L. S., Gray, J. A., Kerwin, R. W., 1995. Latent inhibition in drug-naive schizophrenics: relation-ship to duration of 
illness and dopamine D2 binding using SPET. Schizophr. Res. 17, 95-107.

Gray, N. S., Pickering, A. D., Gray, J. A., Jones, S. H., Abrahams, S. H., Hemsley, D. R., 1997. Kamin blocking is not disrupted by amphetamine in human subjects. J. Psychopharmacology 11, 301-311.

Heim, G., 1989. Attentional functions in listening and schizophrenia: a selective review. Eur. Arch. Psychiatry. Neurol. Sci. 239, 62-69.

Hemsley, D. R., 1987. An experimental psychological model for schizophrenia. In: Häfner, H. Gattaz, W. F., Janzarik, W. (Eds.). Search for the causes of schizophrenia, Springer-Verlag, Berlin, pp. 179-188.

Hoff, A. L., Harris, D., Faustman, W. O., Beal, M., De Villiers, D., Mone, R. D., Moses, J. A., Csernansky, J. G., 1996. A neuropsychological study of early onset schizophrenia. Schizophr. Res. 20, 21-28.

Jacobsen, L. K., Rapoport, J. L. (1998). Childhood-onset schizophrenia: implications of clinical and neurobiological research. J. Child Psychol. Psychiatry 39, 101-113.

Jahanshahi, M. and Frith, C. D., 1999. Willed action and its impairments. Cog. Neuropsychology 15, 483-534.

Jeste, D. V., McAdams, L. A., Palmer, B. W., Braff, D. L., Jernigan, T. L., Stout, J. C., Symonds, L. L., Bailey, A., Heaton, R. K., 1998. Relationship of neuropsychological and MRI measures to age of onset of schizophrenia. Acta Psychiat. Scand. 98, 156-164.

Jones, S. H., Gray, J. A., Hemsley, D. R., 1990. The Kamin blocking effect, incidental learning and psychoticism. Br. J. Psychology 81, 95-110.

Jones, S. H., Gray, J. A., Hemsley, D. R., 1992. The loss of the Kamin blocking effect in acute but not chronic schizophrenics. Biol. Psychiatry 32, 739-755.

Jones, S. H., Hemsley, D. R., Ball, S., Serra, A., 1997. Disruption of the Kamin blocking effect in schizophrenia and in normal subjects following amphetamine. Behav. Brain Res. 88, 103-114, 1997.

Kahlbaum, K. 1863. Die Gruppierung der psychischen Krankheiten. Kafemann, Danzig.
Kamin, L. J., 1969. Predictability, surprise, attention and conditioning. In: R. Church, R., Campbell, B. (Ed.). Punishment and aversive behavior, Appleton-CenturyCrofts, New York, pp. 279-296.

Kay, S. R., Opler, L. A., Fizbein, A., 1992. The Positive and Negative Syndrome Scale (PANSS). Manual. Multi-Health Systems, Toronto.

Lacroix, L., Broersen, L. M., Weiner, I., Feldon, J., 1998. The effects of excitotoxic lesion of the medial prefrontal cortex on latent inhibition, prepulse inhibition, foodhoarding, elevated plus-maze, active avoidance and locomotor activity in the rat. Neuroscience 84, 431-442.

Liddle, P. F., 1987. Schizophrenic syndromes, cognitive performance and neurological dysfunction. Psychol. Med. 17, 49-57.

Lorden, J. F., Rickert, E. J., Pellymounter, M. A., 1980. Forebrain norepinephrine and the selective processing of information. Brain Res. 190, 569-573.

Mackintosh, N.J., 1975. A theory of attention: variations in the associability of stimuli with reinforcement. Psychol. Rev. 82, 276-298.

Magaro, P. A., 1980. Cognition in schizophrenia and paranoia: the integration of cognitive processes. Erlbaum, Hillsdale, NJ.

Nestor, P.G., Shenton, M. E., Wible, C., Hokama, H., O'Donnell, B.F., Law, S., McCarley, R. W. (1998). A neuropsychological analysis of schizophrenic thought disorder. Schizophr. Res. 29, 217-225.

Oades, R. D., Bunk, D., Eggers, C., 1992. Paranoid schizophrenics may not use irrelevant signals: the use of measures of blocking and of urinary dopamine. Acta Paedopsychiatr. 55, 183-184.

Oades, R. D., Röpcke, B., Schepker, R., 1996a. $A$ test of conditioned blocking and its development in childhood and adolescence: relationship to personality and monoamine metabolism. Dev. Neuropsychology 12, 207-230.

Oades, R. D., Zimmermann, B., Eggers, C., 1996b. Conditioned blocking in patients with paranoid, non-paranoid psychosis or obsessive compulsive disorder: associations with symptoms, personality and 
monoamine metabolism. J. Psychiatr. Res. 30, 369-390.

Oades, R. D., Heeper, C., Czwenk, A., Eisenbeis, I., Fierro, L., Dolberg, A., Müller, B. W., What neuropsychological signs are associated with the presence or absence of conditioned blocking in schizophrenia ? Schizophr. Res., 41, 291.

Oades, R. D., Rivet, J-M., Taghzouti, K., Kharouby, M., Simon, H., Le Moal, M., 1987. Catecholamines and conditioned blocking: effects of ventral tegmental, septal and frontal 6-hydroxydopamine lesions in rats. Brain Res. 406, 136-146.

Oldfield, R. C., 1971. The assessment and analysis of handedness: The Edinburgh inventory. Neuropsychologia 9, 97-113.

Rickert, E. J., Bennett, T. L., Lane, P. and French, J., 1978. Hippocampectomy and the attenuation of blocking. Behav. Biol. 22, 147-160.

Simpson, G. M., Angus, J. W. S., 1970. A rating scale for extrapyramidal side-effects. Acta Psychiatr. Scand. supplement 212, 11-19.

Solomon, P. R., 1977. Role of the hippocampus in blocking and conditioned inhibition of the rat's nictitating membrane response. J. Comp. Physiol. Psychology 91, 407-417.

Stirling, J. D., Hellewell, J. S. E., Qurashi, N., (1998). Self-monitoring dysfunction and the schizophrenic symptoms of alien control. Psychol. Med. 28, 675-683.

Weisbrod, M., Maier, S., Harig, S., Himmelsbach, U., Spitzer, M., (1998). Lateralized semantic and indirect semantic priming effects in people with schizophrenia. Br. J. Psychiatry 172, 142146. 